\title{
Finite volume element approximation of an inhomogeneous Brusselator model with cross-diffusion
}

\author{
Zhigui Lin ${ }^{\mathrm{a}}$, Ricardo Ruiz-Baier ${ }^{\mathrm{b}, 1}$, Canrong Tian ${ }^{\mathrm{c}, *}$ \\ a School of Mathematical Science, Yangzhou University, Yangzhou 225002, China \\ b Modeling and Scientific Computing, MATHICSE-SB, École Polytechnique Fédérale de Lausanne, CH-1015 Lausanne, Switzerland \\ c Department of Basic Sciences, Yancheng Institute of Technology, Yancheng 224003, China
}

\section{A R T I C L E I N F O}

\section{Article history:}

Received 24 August 2012

Received in revised form 31 August 2013

Accepted 5 September 2013

Available online 13 September 2013

\section{Keywords:}

Finite volume element method

Brusselator model

Cross-diffusion effect

Spatial patterns

\begin{abstract}
A B S T R A C T
This paper is concerned with the study of pattern formation for an inhomogeneous Brusselator model with cross-diffusion, modeling an autocatalytic chemical reaction taking place in a three-dimensional domain. For the spatial discretization of the problem we develop a novel finite volume element (FVE) method associated to a piecewise linear finite element approximation of the cross-diffusion system. We study the main properties of the unique equilibrium of the related dynamical system. A rigorous linear stability analysis around the spatially homogeneous steady state is provided and we address in detail the formation of Turing patterns driven by the cross-diffusion effect. In addition we focus on the spatial accuracy of the FVE method, and a series of numerical simulations confirm the expected behavior of the solutions. In particular we show that, depending on the spatial dimension, the magnitude of the cross-diffusion influences the selection of spatial patterns.
\end{abstract}

(c) 2013 Elsevier Inc. All rights reserved.

\section{Introduction}

The theory of spatial patterns generation goes back to the pioneering work of Turing [46]. Essentially, one chemical, the activator, stimulated and enhanced the production of the other chemical, which, in turn, depleted or inhibited the formation of the activator. The so-called Turing mechanism of pattern formation is onset by a diffusion-induced instability around the homogeneous steady state, that is, the concentration of the species evolves from an initial near homogeneity into inhomogeneous spatial distributions. This typically occurs if the diffusion of the inhibitor is large enough in comparison to that of the activator. This phenomenon has been reported in the context of the chlorite-iodide-malonic acid (CIMA) reaction [12,35] (see also [15]). On the other hand, further experimental studies have demonstrated that the cross-diffusion effect can lead to the formation of spatial and spatiotemporal patterns (see e.g. [47]). This is an interesting phenomenon whose applications range from biochemical to physical and economical processes. Numerous investigations from the viewpoint of mathematical and numerical analysis deal with some aspects of these problems, mainly focusing on the one- or two-dimensional case. In [4], it is shown that the spatial dimension has an important influence on the Turing pattern behavior. In the case when the wavelength of the Turing pattern is sufficiently (at least two times) larger than the thickness of the medium, threedimensional patterns can be simplified to two-dimensional patterns. Otherwise, the three-dimensional patterns may differ from the well studied two-dimensional case (see $[28,30]$ and the references therein). In this paper we aim at studying to which extent the spatial dimension influences the pattern behavior. Our analysis differs from the one in [4] in that we considered Turing patterns affected/induced by cross-diffusion mechanisms. We will focus on an inhomogeneous Brusselator model (see e.g. [21,36]), here assuming the following form

\footnotetext{
* Corresponding author. Tel.: +86 051588168221.

E-mail addresses: zglin68@hotmail.com (Z. Lin), ricardo.ruiz@epfl.ch (R. Ruiz-Baier), tcr_2001@sohu.com (C. Tian).

1 Present address: CRET-FGSE, University of Lausanne, UNIL-Mouline, CH-1015, Lausanne, Switzerland.
} 


$$
\begin{cases}\frac{\partial u_{1}}{\partial t}-\Delta\left(D_{11} u_{1}+D_{12} u_{2}\right)=-(\beta+1) u_{1}+u_{1}^{2} u_{2}+\alpha, & (x, t) \in \Omega_{T}, \\ \frac{\partial u_{2}}{\partial t}-\Delta\left(D_{21} u_{1}+D_{22} u_{2}\right)=\beta u_{1}-u_{1}^{2} u_{2}, & (x, t) \in \Omega_{T}, \\ \frac{\partial u_{1}}{\partial \eta}=\frac{\partial u_{2}}{\partial \eta}=0, & (x, t) \in \Sigma_{T}, \\ u_{1}(x, 0)=\psi_{1}(x), \quad u_{2}(x, 0)=\psi_{2}(x), & x \in \Omega,\end{cases}
$$

where $\Omega_{T}:=\Omega \times(0, T), \Sigma_{T}:=(\partial \Omega) \times(0, T)$ for a fixed $T>0$. We take $\alpha$ and $\beta$ as positive constants, whereas $D_{11}$ and $D_{22}$ are the self-diffusion coefficients. The term $\Delta\left(D_{i j} u_{j}\right)=\nabla \cdot\left(\nabla\left(D_{i j} u_{j}\right)\right)$ takes into account the flux of $u_{i}, \nabla\left(D_{i j} u_{j}\right)$, induced by the gradient of species $u_{j}$. Likewise, $D_{i j}$ is the cross-diffusion coefficient for $i \neq j$. This system arises in the mathematical modeling of an autocatalytic chemical interaction governed by the following reaction mechanism:

$$
A \stackrel{k_{1}}{\rightarrow} X, \quad B+X \stackrel{k_{2}}{\longrightarrow} Y+D, \quad 2 X+Y \stackrel{k_{3}}{\longrightarrow} 3 X, \quad X \stackrel{k_{4}}{\rightarrow} \text { Products. }
$$

Here $A$ and $B$ are the major species, $X$ and $Y$ the intermediate species. The third step is autocatalytic. As in [9], after employing the following scaled variables

$$
\alpha=\left(\frac{k_{1}^{2} k_{3}}{k_{4}^{3}}\right)^{\frac{1}{2}} A, \quad \beta=\left(\frac{k_{2}}{k_{4}}\right) B, \quad u_{1}=\left(\frac{k_{3}}{k_{4}}\right)^{\frac{1}{2}} X, \quad u_{2}=\left(\frac{k_{3}}{k_{4}}\right)^{\frac{1}{2}} Y, \quad \bar{t}=k_{4} t,
$$

and dropping the bar on $t$, we find that the evolution of $u_{1}$ and $u_{2}$ is governed by the ODE system

$$
\left\{\begin{array}{l}
\frac{d u_{1}}{d t}=-(\beta+1) u_{1}+u_{1}^{2} u_{2}+\alpha \\
\frac{d u_{2}}{d t}=\beta u_{1}-u_{1}^{2} u_{2}
\end{array}\right.
$$

By introducing the cross-diffusion effect as in [47], system (1.2) leads to (1.1). This system is a suitable prototype for the study of a larger class of reaction-diffusion systems. By means of a linearized stability analysis, we will first show that if the parameters satisfy the condition $\beta<\min \left\{1+\alpha^{2}, 1+\frac{\alpha^{2} D_{11}-2 \alpha \sqrt{D_{11} D_{22}}}{D_{22}}\right\}$, then the cross-diffusion effect gives rise to the formation of patterns. Then, we focus on the spatial structure of these patterns with the help of a series of numerical tests.

An important number of contributions have been proposed to treat systems like (1.1) from a numerical perspective, either considering or not the cross-diffusion effect (see $[2,5,6,8,14,18,21,39]$ for finite differences, finite volumes, spectral and finite element methods for the spatial discretization). Here, and following [11,27,37], we propose a new finite volume element (FVE) method for the numerical approximation of the underlying reaction-cross-diffusion system. We do not intend to carry out a thorough comparison of the performances of the different discretization strategies, but we rather introduce a FVE formulation because of its natural mass conservation property, and we employ it to study the formation and identification of spatial patterns.

FVE methods exhibit several advantages over some of the approaches mentioned above. These include the ability of treating arbitrarily complex geometries, unstructured and anisotropic meshes, a variety of boundary conditions (as robust finite element methods) and they feature local conservation and front capturing properties inherent mainly to finite volume methods (and highly desirable in the simulation of population dynamics). The key idea is that a complementary dual (or adjoint) mesh is introduced and a transfer map permits to rewrite a classical Galerkin formulation as a finite volume method (that is, in terms of fluxes passing through the faces of the primal elements). In the end, it is possible to reformulate the discrete problem as a Petrov-Galerkin problem. Related variants are also known as marker and cell methods [20], generalized difference methods [29], finite volume methods [1], covolume methods [34], box methods [3] or combined finite volume-finite element methods [24]. These are in general restricted to the two-dimensional case. We stress that even if in the present contribution we propose a FVE method for the particular inhomogeneous Brusselator system (1.1), the derivation of the FVE formulation is suitable for a larger class of Turing-type models including e.g. the well-known Gray-Scott [22], Gierer-Meinhardt [19] or the Schnakenberg [41] equations. In addition, the convergence properties of FVE methods can be studied rather straightforwardly by recasting the discrete formulation in a classical abstract framework for nonlinear Petrov-Galerkin problems. Even if a rigorous convergence analysis goes beyond the scope of the paper, we stress that optimal experimental rates of convergence are observed for both species, in the sense that the observed errors exhibit the same convergence order as the finite element interpolation operators.

The remainder of this paper is structured as follows. In Section 2 we deduce from the mathematical standpoint, the role of cross-diffusion in the generation of spatial patterns, and we provide the conditions for these patterns to appear. A FVE formulation for approximating the governing equations is detailed in Section 3, and some numerical tests including the study of convergence and formation of spatial patterns are shown in Section 4. Finally, some conclusions are drawn in Section 5. Proofs of our main results are collected in Appendices A, B and C. 


\section{Turing patterns driven by cross-diffusion effects}

In this section, we aim to explore the effect of the cross-diffusion on the stationary structure of Brusselator model. We will first find the conditions under which the positive equilibrium is linearly stable without cross-diffusion but unstable with cross-diffusion, a well-known phenomenon of cross-diffusion driven instability (see similar studies in [21,43-45]). We will start by determining the bifurcation point, and then we will investigate the properties of the associated Turing patterns.

\subsection{Linear stability analysis}

The existence and local stability of an internal equilibrium point $\mathbf{u}^{*}:=\left(u_{1}^{*}, u_{2}^{*}\right)$ where the two species coexist, depends on the parameter values. For system (1.1), there is a unique positive equilibrium point $\left(u_{1}^{*}, u_{2}^{*}\right)=(\alpha, \beta / \alpha)$ for any positive constants $\alpha$ and $\beta$. We now analyze the local asymptotic stability of the positive equilibrium to (1.1).

Throughout this paper we will denote

$$
\mathbf{G}(\mathbf{u})=\left(\begin{array}{l}
G_{1}(\mathbf{u}) \\
G_{2}(\mathbf{u})
\end{array}\right)=\left(\begin{array}{c}
-(\beta+1) u_{1}+u_{1}^{2} u_{2}+\alpha \\
\beta u_{1}-u_{1}^{2} u_{2}
\end{array}\right) .
$$

The following stability and instability results hold for the positive equilibrium (the proofs are postponed to Appendices A, B and $C$, respectively).

Theorem 2.1. In the absence of cross-diffusion effects, that is $D_{12}=D_{21}=0$, the positive equilibrium point $\mathbf{u}^{*}$ of (1.1) is locally asymptotic stable if and only if the model parameters satisfy

$$
\beta<\min \left\{1+\alpha^{2}, 1+\frac{\alpha^{2} D_{11}-2 \alpha \sqrt{D_{11} D_{22}}}{D_{22}}\right\} .
$$

Theorem 2.1 suggests that the self-diffusion cannot induce instabilities to the positive equilibrium point if and only if (2.1) is satisfied. Now, we consider the influence of cross-diffusion on the steady states. Namely, we show that when the cross-diffusion is small, the inhomogeneous steady state of (1.1) does not exist.

Theorem 2.2. Assume that $0 \leqslant u_{1}(x, t) \leqslant K_{1}, 0 \leqslant u_{2}(x, t) \leqslant K_{2}$ for all $(x, t) \in \Omega_{T}$. If

$$
D_{11}>\frac{2 K_{1} K_{2}-\beta-1}{\mu_{2}}, \quad \sqrt{D_{22}\left(D_{11}-\frac{2 K_{1} K_{2}-\beta-1}{\mu_{2}}\right)}>\frac{1}{2}\left(D_{12}+D_{21}+\frac{K_{1}^{2}+2 K_{1} K_{2}+\beta}{\mu_{2}}\right),
$$

then the system (1.1) has no inhomogeneous steady state.

The above theorem implies that Turing patterns cannot occur provided that the cross-diffusion is small. In what follows we will find necessary and sufficient conditions for Turing patterns to occur.

Theorem 2.3. Assume that condition (2.1) holds. Then the equilibrium $\mathbf{u}^{*}$ of (1.1) is unstable if and only if

$$
D_{12} \geqslant \frac{D_{11} D_{22}}{D_{21}} \text { or } \frac{1}{\beta}\left[\alpha^{2}\left(D_{11}+D_{21}\right)+(1-\beta) D_{22}+2 \alpha \sqrt{D_{11} D_{22}-D_{12} D_{21}}\right] \leqslant D_{12}<\frac{D_{11} D_{22}}{D_{21}} \text {. }
$$

The above theorems reveal that the cross-diffusion effect is able to destabilize the positive equilibrium, and result in Turing patterns.

\subsection{Turing parameter space}

In view of Theorem 2.3 , the fulfillment of the following conditions is sufficient for the positive equilibrium point $\left(u_{1}^{*}, u_{2}^{*}\right)$ being linearly unstable with respect to the particular case of system (1.1):

(i) $\beta<\min \left\{1+\alpha^{2}, 1+\frac{\alpha^{2} D_{11}-2 \alpha \sqrt{D_{11} D_{22}}}{D_{22}}\right\}$.

(ii) $\frac{1}{\beta}\left[\alpha^{2}\left(D_{11}+D_{21}\right)+(1-\beta) D_{22}+2 \alpha \sqrt{D_{11} D_{22}-D_{12} D_{21}}\right] \leqslant D_{12}$.

In the remainder of the paper, the values satisfying the parameter spaces will be set as follows:

$$
\alpha=6, \quad \beta=1, \quad D_{11}=0.4, \quad D_{22}=2, \quad D_{21}=0.02 .
$$

For this particular choice, the positive stationary uniform solution is given by 


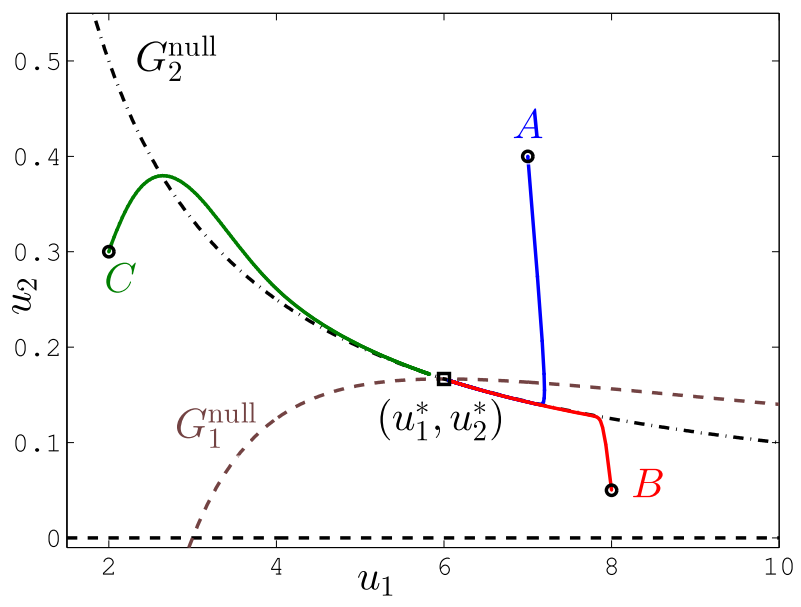

Fig. 1. Phase plane for model (1.1). Computed trajectories starting from states $A(7,0.4), B(8,0.05), C(2,0.3)$ and reaching the equilibrium (2.4).

$$
\left(u_{1}^{*}, u_{2}^{*}\right)=(6,0.1667) .
$$

If no diffusion is considered, problem (1.1) boils down to the dynamical system

$$
\frac{d \mathbf{u}}{d t}=\mathbf{G}(\mathbf{u}),
$$

whose phase diagram is presented in Fig. 1 . We show computed trajectories for different initial values of $u_{1}(x, 0)$ and $u_{2}(x, 0)$ which converge to the equilibrium state $(2.4)$, given by the intersection of the (nontrivial) nullclines

$$
G_{1}^{\text {null }}\left(u_{1}\right)=\frac{(\beta+1) u_{1}-\alpha}{u_{1}^{2}}, \quad G_{2}^{\text {null }}\left(u_{1}\right)=\frac{\beta}{u_{1}} .
$$

As the similar method in [33], we are able to calculate the wavenumber explicitly and determine the pattern selection by linearizing cross-diffusion around the stationary uniform solution and taking $D_{12}$ as the Turing bifurcation parameter. The Turing bifurcation occurs when $\operatorname{Re}(\lambda(\mathbf{k}))=0$ at $\mathbf{k}=\mathbf{k}_{c} \neq 0$, where $\mathbf{k}_{c}$ is the critical wavenumber and $|\mathbf{k}|^{2}$ is equivalent to the eigenvalue $\mu_{i}$. From Theorem 2.3, we find that the Turing bifurcation threshold satisfies

$$
D_{12}=\frac{1}{\beta}\left[\alpha^{2}\left(D_{11}+D_{21}\right)+(1-\beta) D_{22}+2 \alpha \sqrt{D_{11} D_{22}-D_{12} D_{21}}\right] .
$$

Moreover, we have the following wavenumber:

$$
\left|\mathbf{k}_{c}\right|= \begin{cases}\sqrt{\frac{-\alpha^{2} D_{11}-(1-\beta) D_{22}+\beta D_{12}-\alpha^{2} D_{21}}{2\left(D_{11} D_{22}-D_{12} D_{21}\right)},}, & \text { if } D_{12}<\frac{D_{11} D_{22}}{D_{21}}, \\ \sqrt{\frac{\alpha^{2} D_{11}+(1-\beta) D_{22}-\beta D_{12}+\alpha^{2} D_{21}}{2\left(D_{11} D_{22}-D_{12} D_{21}\right)},}, & \text { otherwise. }\end{cases}
$$

\section{Finite volume element approximation}

\subsection{Weak form of the cross-diffusion system}

Multiplying the first two equations of (1.1) by smooth functions $v$, and $w$, respectively, and integrating by parts over $\Omega$ yield the following weak formulation of the cross-diffusion system: For $0<t<T$, find $u_{1}(t), u_{2}(t) \in H^{1}(\Omega)$ such that

$$
\begin{aligned}
& \frac{\partial}{\partial t} \int_{\Omega} u_{1} v d x+\int_{\Omega}\left(D_{11} \nabla u_{1}+D_{12} \nabla u_{2}\right) \cdot \nabla v d x=\int_{\Omega}\left(-(\beta+1) u_{1}+u_{1}^{2} u_{2}+\alpha\right) v d x, \quad \forall v \in H^{1}(\Omega), \\
& \frac{\partial}{\partial t} \int_{\Omega} u_{2} w d x+\int_{\Omega}\left(D_{21} \nabla u_{1}+D_{22} \nabla u_{2}\right) \cdot \nabla w d x=\int_{\Omega}\left(\beta u_{1}-u_{1}^{2} u_{2}\right) w d x, \quad \forall w \in H^{1}(\Omega),
\end{aligned}
$$

and $u_{1}(0)=u_{1,0}, u_{2}(0)=u_{2,0}$ a.e. in $\Omega$. The existence of weak solutions for a class of reaction-diffusion systems covering this case has been established in [2,13]. Global existence can be proved using the arguments in e.g. [25]. 

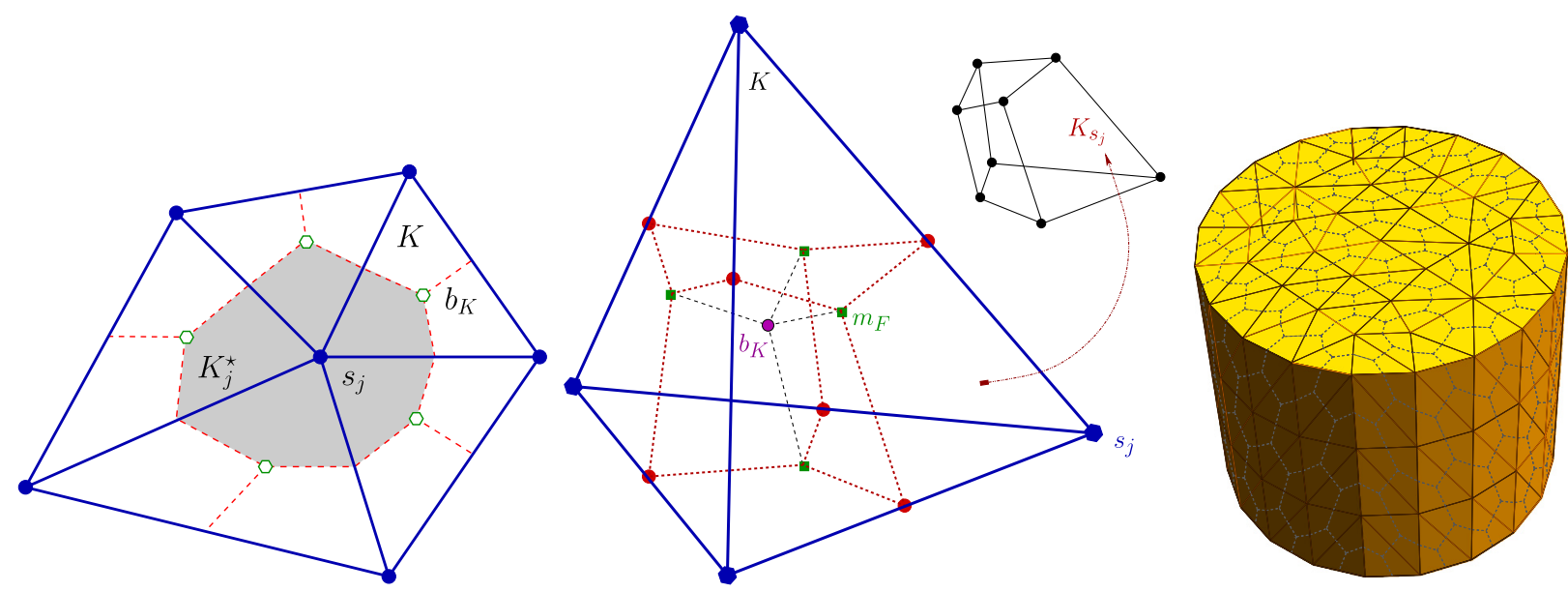

Fig. 2. Sketch of primal and dual meshes, finite elements and control volumes on a local 2D partition (left) and a single primal tetrahedron split into four polyhedra $K_{z}$ (middle). Right: Example of primal and dual meshes (solid and dashed lines, respectively) on a 3D geometry where the primal mesh consists of $\mathcal{N}_{h}=176$ vertices and 594 tetrahedral elements.

\subsection{Spatial discretization}

Let us assume that $\Omega$ is a polyhedral domain and let $\mathcal{T}_{h}$ denote a partition of $\Omega$ into tetrahedral elements $K$ of diameter $h_{K}$ satisfying the property of local regularity: There exists $C>0$ such that

$$
C h_{K}^{2} \leqslant|K| \leqslant h_{K}^{2}
$$

for all $K \in \mathcal{T}_{h}$. The mesh parameter is $h:=\max _{K \in \mathcal{T}_{h}}\left\{h_{K}\right\}$ and $\mathcal{N}_{h}:=\left\{s_{j}: j=1, \ldots, N\right\}$ denotes the set of vertices of $\mathcal{T}_{h}$. By $\mathcal{E}_{h}$ we denote the complete set of faces of $\mathcal{T}_{h}$ and $\mathcal{E}_{h}^{\text {int }}$ is its restriction to the faces not lying on $\partial \Omega$. Let $V_{h}$ denote the standard finite element space of continuous piecewise linear functions to be employed in the approximation of the fields $u_{1}, u_{2}$. That is,

$$
V_{h}:=\left\{v \in H^{1}(\Omega) \cap C^{0}(\bar{\Omega}):\left.v\right|_{K} \in \mathbb{P}_{1}(K) \text { for all } K \in \mathcal{T}_{h}\right\},
$$

where $\mathbb{P}_{m}(K)$ denotes the space of polynomial functions of total degree $s \leqslant m$ defined on the element $K$. A semi-discrete Galerkin finite element method for the approximation of (3.1) reads: For $t \in(0, T]$, find $u_{1, h}(t)$ and $u_{2, h}(t) \in V_{h}$ such that

$$
\begin{aligned}
& \frac{d}{d t}\left(u_{1, h}, v_{h}\right)_{\Omega}+D_{11}\left(\nabla u_{1, h}, \nabla v_{h}\right)_{\Omega}+D_{12}\left(\nabla u_{2, h}, \nabla v_{h}\right)_{\Omega}=\left(f_{h}, v_{h}\right)_{\Omega}, \quad \forall v_{h} \in V_{h}, \\
& \frac{d}{d t}\left(u_{2, h}, w_{h}\right)_{\Omega}+D_{21}\left(\nabla u_{1, h}, \nabla w_{h}\right)_{\Omega}+D_{22}\left(\nabla u_{2, h}, \nabla w_{h}\right)_{\Omega}=\left(g_{h}, w_{h}\right)_{\Omega}, \quad \forall w_{h} \in V_{h},
\end{aligned}
$$

where $(\cdot, \cdot)_{\Omega}$ denotes the inner product in $L^{2}(\Omega)$ and $f_{h}=-(\beta+1) u_{1, h}+u_{1, h}^{2} u_{2, h}+\alpha, g_{h}=\beta u_{1, h}-u_{1, h}^{2} u_{2, h}$. Under stronger regularity requirements on $u_{1}, u_{2}$ it is possible to show that the fully discrete Galerkin scheme is well-posed and convergent (see [5]). The approximate initial concentrations $u_{1, h}(0), u_{2, h}(0)$ are constructed as the $L^{2}$ projections of $u_{1,0}$ and $u_{2,0}$ onto $V_{h}$.

In order to define a FVE formulation associated to (3.2) we introduce a median-based tessellation of $\Omega$ formed by joining the median dual segments of each tetrahedron in $\mathcal{T}_{h}$. Such partition is called dual mesh and its denoted by $\mathcal{T}_{h}^{\star}$. For each element $K \in \mathcal{T}_{h}$ we create segments joining its barycenter $b_{K}$ with the midpoints (2D barycenters) $m_{F}$ of each face $F \subset \partial K$, forming four polyhedra $K_{z}$ for $z$ in the set of vertices of $K$, that is, $z \in \mathcal{N}_{h} \cap K$. Then to each vertex $s_{j} \in \mathcal{N}_{h}$, we associate a so-called control volume $K_{j}^{\star}$ consisting of the union of the polyhedra $K_{s_{j}}$ sharing the vertex $s_{j}$ (see Fig. 2). We now define the following finite-dimensional space associated to the dual partition $\mathcal{T}_{h}^{\star}$ (which is also quasi-uniform):

$$
V_{h}^{\star}:=\left\{v \in L^{2}(\Omega):\left.v\right|_{K_{j}^{\star}} \in \mathbb{P}_{0}\left(K_{j}^{\star}\right) \text { for all } K_{j}^{\star} \in \mathcal{T}_{h}^{\star}\right\} .
$$

The primal and dual meshes are related by the transfer operator $\mathcal{P}: V_{h} \rightarrow V_{h}^{\star}$ (see e.g. [37]) defined by

$$
\left(\mathcal{P}_{h} v_{h}\right)(x)=\sum_{j=1}^{\mathcal{N}_{h}} v_{h}\left(s_{j}\right) \chi_{j}(x) \quad \text { for } x \in \Omega,
$$


where $\chi_{j}$ is the characteristic function on the control volume $K_{j}^{\star}$. To derive the FVE formulation we multiply the first two equations in (1.1) by $\mathcal{P}_{h} v_{h} \in V_{h}^{\star}$ and $\mathcal{P}_{h} w_{h} \in V_{h}^{\star}$, then integrate by parts over each $K_{j}^{\star} \in \mathcal{T}_{h}^{\star}$. We get: For $t \in(0, T]$, find $u_{1, h}(t), u_{2, h}(t) \in V_{h}$ such that

$$
\begin{aligned}
& \frac{d}{d t}\left(u_{1, h}, \mathcal{P}_{h} v_{h}\right)_{\Omega}-D_{11} \sum_{j=1}^{\mathcal{N}_{h}} v_{h}\left(s_{j}\right) \int_{\partial K_{j}^{\star}} \nabla u_{1, h} \cdot \mathbf{n}-D_{12} \sum_{j=1}^{\mathcal{N}_{h}} v_{h}\left(s_{j}\right) \int_{\partial K_{j}^{\star}} \nabla u_{2, h} \cdot \mathbf{n}=\left(f_{h}, \mathcal{P}_{h} v_{h}\right)_{\Omega}, \quad \forall v_{h} \in V_{h}, \\
& \frac{d}{d t}\left(u_{2, h}, \mathcal{P}_{h} w_{h}\right)_{\Omega}-D_{21} \sum_{j=1}^{\mathcal{N}_{h}} w_{h}\left(s_{j}\right) \int_{\partial K_{j}^{\star}} \nabla u_{1, h} \cdot \mathbf{n}-D_{22} \sum_{j=1}^{\mathcal{N}_{h}} w_{h}\left(s_{j}\right) \int_{\partial K_{j}^{\star}} \nabla u_{2, h} \cdot \mathbf{n}=\left(g_{h}, \mathcal{P}_{h} w_{h}\right)_{\Omega}, \quad \forall w_{h} \in V_{h}, \\
& u_{1, h}(0)=\mathcal{R}_{h} u_{1,0}, \quad u_{2, h}(0)=\mathcal{R}_{h} u_{2,0},
\end{aligned}
$$

where $\mathcal{R}_{h}: H^{2}(\Omega) \rightarrow V_{h}$ is a projection defined by the diffusion operator as follows

$$
\sum_{j=1}^{\mathcal{N}_{h}} v_{h}\left(s_{j}\right) \int_{\partial K_{j}^{\star}} \nabla\left(u_{i}-\mathcal{R}_{h} u_{i}\right) \cdot \mathbf{n}=0, \quad \forall v_{h} \in V_{h}, i=1,2 .
$$

The proof of the following lemma can be found in e.g. [27].

Lemma 3.1. For any $v_{h}, w_{h} \in V_{h}$, the following relation holds

$$
-\sum_{j=1}^{\mathcal{N}_{h}} w_{h}\left(s_{j}\right) \int_{\partial K_{j}^{\star}} \nabla v_{h} \cdot \mathbf{n}=\left(\nabla v_{h}, \nabla w_{h}\right)_{\Omega}
$$

This allows us to recast the previous finite volume method as: For $t \in(0, T]$, find $u_{1, h}(t), u_{2, h}(t) \in V_{h}$ such that

$$
\begin{aligned}
& \frac{d}{d t}\left(u_{1, h}, v_{h}\right)_{\Omega}+D_{11}\left(\nabla u_{1, h}, \nabla v_{h}\right)_{\Omega}+D_{12}\left(\nabla u_{2, h}, \nabla v_{h}\right)_{\Omega}=\left(f_{h}, \mathcal{P}_{h} v_{h}\right)_{\Omega}, \quad \forall v_{h} \in V_{h}, \\
& \frac{d}{d t}\left(u_{2, h}, w_{h}\right)_{\Omega}+D_{21}\left(\nabla u_{1, h}, \nabla w_{h}\right)_{\Omega}+D_{22}\left(\nabla u_{2, h}, \nabla w_{h}\right)_{\Omega}=\left(g_{h}, \mathcal{P}_{h} w_{h}\right)_{\Omega}, \quad \forall w_{h} \in V_{h} .
\end{aligned}
$$

\subsection{Temporal discretization}

The evolution in time is performed considering a uniform partition of the interval $[0, T]$ as $\left[0, \ldots, t^{n}, \ldots, T\right]$ into subintervals of size $\Delta t$. Suitable time integration strategies include Runge-Kutta schemes as in [32,48], Crank-Nicholson methods [17,42] or local time-stepping [7]. Here we opt for a second order backward difference advancing scheme (BDF2, see e.g. [23]). The space-time discrete FVE method then reads: For $n \geqslant 1$, given $u_{1, h}^{n}$ and $u_{2, h}^{n}$, find $\left(u_{1, h}^{n+1}\right.$, $\left.u_{2, h}^{n+1}\right)$ such that for all $v_{h}, w_{h} \in V_{h}$ it holds

$$
\begin{aligned}
& \left(\tilde{u}_{1, h}^{n+1}, v_{h}\right)_{\Omega}+D_{11}\left(\nabla u_{1, h}^{n+1}, \nabla v_{h}\right)_{\Omega}+D_{12}\left(\nabla u_{2, h}^{n+1}, \nabla v_{h}\right)_{\Omega}=\left(f_{h}^{n}, \mathcal{P}_{h} v_{h}\right)_{\Omega}, \\
& \left(\tilde{u}_{2, h}^{n+1}, w_{h}\right)_{\Omega}+D_{21}\left(\nabla u_{1, h}^{n+1}, \nabla w_{h}\right)_{\Omega}+D_{22}\left(\nabla u_{2, h}^{n+1}, \nabla w_{h}\right)_{\Omega}=\left(g_{h}^{n}, \mathcal{P}_{h} w_{h}\right)_{\Omega}
\end{aligned}
$$

for all $v_{h}, w_{h} \in V_{h}$, where

$$
\tilde{u}_{i, h}^{n+1}:=\frac{1}{\Delta t}\left(\frac{3}{2} u_{i, h}^{n+1}-2 u_{i, h}^{n}+\frac{1}{2} u_{i, h}^{n-1}\right), \quad i=1,2,
$$

and the reaction terms are discretized in an explicit manner, i.e. $f_{h}^{n}=-(\beta+1) u_{1, h}^{n}+\left(u_{1, h}^{n}\right)^{2} u_{2, h}^{n}+\alpha, g_{h}=\beta u_{1, h}^{n}-\left(u_{1, h}^{n}\right)^{2} u_{2, h}^{n}$. In matrix form, (3.3) boils down to

$$
\mathbf{M} \tilde{\mathbf{U}}^{n+1}+\mathbf{K} \mathbf{U}^{n+1}=(\mathbb{P} \mathbf{F}) \mathbf{U}^{n}
$$

where $\mathbf{U}$ is the total solution vector, $\tilde{\mathbf{U}}$ is the approximation of the time derivative of $\mathbf{U}$, and $\mathbf{M}, \mathbf{K}, \mathbb{P}, \mathbf{F}$ are the usual mass, stiffness, primal-dual projection and reaction matrices, respectively. At each time iteration the resulting linear system is solved by means of the GMRES method (a tolerance of $1 \mathrm{e}-7$ is used for the $L^{2}$-norm of the residual), combined with Schwarz preconditioning (see e.g. [40]). 

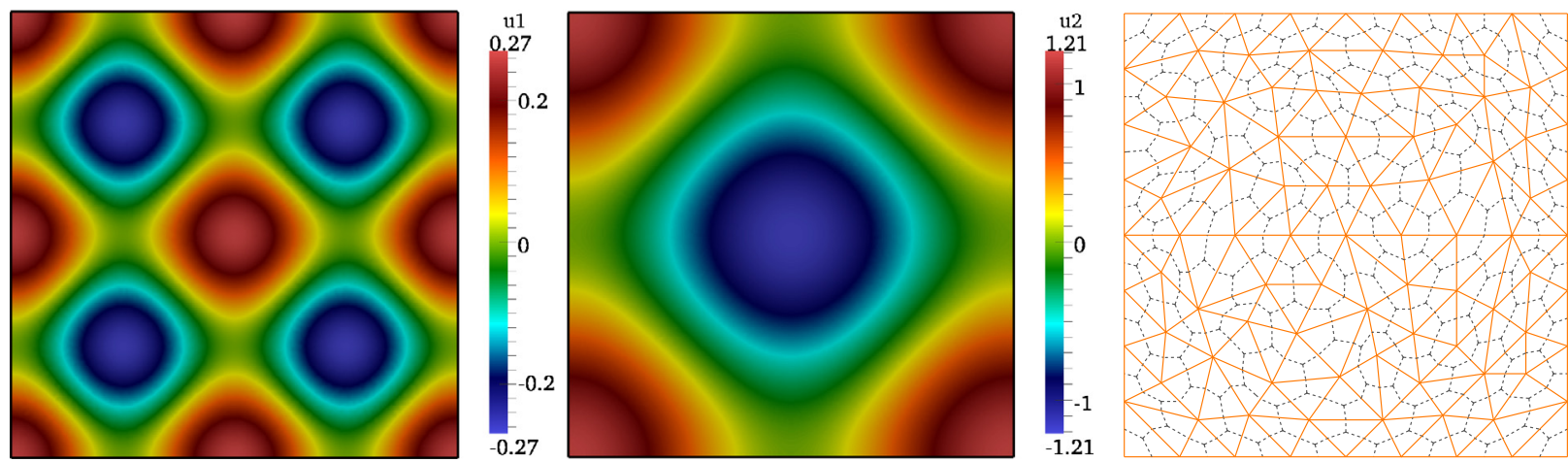

Fig. 3. Validation test: Finite volume element approximations of the fields $u_{1}$ and $u_{2}$ at the final time $T=0.5$ (left and middle, respectively), and example of a primal and dual mesh.

\section{Numerical results}

In this section we present results of computational examples using the finite volume element method. Unless otherwise explicitly specified, all units are considered adimensional.

\subsection{Validation test}

As numerical validation of the spatial accuracy of our method we first consider a simple auxiliary reaction-diffusion system given by

$$
\begin{aligned}
& \frac{\partial u_{1}}{\partial t}-\Delta\left(D_{11} u_{1}+D_{12} u_{2}\right)=D_{12} u_{2}, \quad \frac{\partial u_{2}}{\partial t}-\Delta\left(D_{21} u_{1}+D_{22} u_{2}\right)=4 D_{21} u_{1}, \\
& u_{1}(x, y, 0)=\cos (2 x)+\cos (2 y), \quad u_{2}(x, y, 0)=\cos (x)+\cos (y),
\end{aligned}
$$

defined on the square $(0,2 \pi)^{2}$, and where $D_{11}=D_{22}=1, D_{12}=1.5, D_{21}=0.5$. The exact solution is

$$
u_{1}(x, y, t)=\exp (-4 t)(\cos (2 x)+\cos (2 y)), \quad u_{2}(x, y, t)=\exp (-t)(\cos (x)+\cos (y)),
$$

and zero-flux boundary conditions are used. We evolve system (4.1) until $T=1 / 2$ and the approximate solutions are plotted in Fig. 3. At a fixed time instant $\tilde{t}$ we compute errors and observed convergence rates defined as

$$
e_{m}(u):=\frac{\left|u-u_{h}\right|_{m, \Omega}}{|u|_{m, \Omega}}, \quad r_{m}(u):=\frac{\log \left(e_{m}(u) / \hat{e}_{m}(u)\right)}{\log (h / \hat{h})},
$$

with $m \in\{0,1\}$, where $e_{m}$ and $\hat{e}_{m}$ are errors obtained for two consecutively refined meshes of sizes $h$ and $\hat{h}$. The spatial convergence is assessed by considering successively refined primal unstructured meshes of 9, 27, 96, 333, 1265 and 4972 vertices. The error history is displayed in Table 1 and Fig. 4, where we observe convergence rates of $\mathcal{O}(h)$ for $u_{1}, u_{2}$ in the $H^{1}$-seminorm and of $\mathcal{O}\left(h^{2}\right)$ in the $L^{2}$-norm. These rates are in agreement with theoretical results from e.g. [16].

In what follows we provide a set of numerical tests to study the different patterns arising from (1.1). The computational domain is now confined to the cube $\Omega=(0, L)^{3}$. The wavenumber satisfies

$$
\mathbf{k}=\pi\left(m_{1} / L, m_{2} / L, m_{3} / L\right), \quad|\mathbf{k}|=\pi \sqrt{\left(m_{1} / L\right)^{2}+\left(m_{2} / L\right)^{2}+\left(m_{3} / L\right)^{2}}, \quad m_{1}, m_{2}, m_{3}=0,1, \ldots
$$

and from (2.5) we obtain the Turing bifurcation threshold $D_{12}=22.2665$. Fig. 5(left) depicts the real part of the eigenvalues $\operatorname{Re}(\lambda)$ versus the norm of the wave vector.

The domain size $L$ is chosen large enough to include several spatial wavelengths. In contrast with ODE-based models, our results indicate that the spatial dynamics is little affected by initial data (see also [31,49]). The initial solutions are chosen as small random perturbations of the equilibrium, that is, we set $u_{1}(\boldsymbol{x}, 0)=\frac{\operatorname{rand}(\boldsymbol{x})}{3}+5.8, u_{2}(\boldsymbol{x}, 0)=\frac{\operatorname{rand}(\boldsymbol{x})}{10}+0.13$, for $\boldsymbol{x} \in \Omega$, where rand $\Omega \rightarrow[0,1]$ is a uniform random distribution. Homogeneous Neumann conditions are imposed on all boundaries. A parallel implementation was performed, specially needed for the three-dimensional tests on fine meshes and for long time horizons. Our $\mathrm{C}++$ code is based on the Trilinos library ${ }^{2}$ and employs OpenMPI for the parallel communications and the Parmetis library [26] for creating the partition of $\mathcal{T}_{h}$ (see Fig. 5(right)). We typically use 16 subdomains and run the simulations on 16 dual-processor nodes, assigning each subdomain to a unique node. The preconditioner is parallelized with

\footnotetext{
2 http://www.trilinos.sandia.gov.
} 
Table 1

Validation test: Convergence histories for the finite volume element method applied to (4.1). The displayed quantities correspond to relative errors and convergence rates in the $L^{2}$-norm and $H^{1}$-seminorm for species $u_{1}, u_{2}$ at $T=1$.

\begin{tabular}{|c|c|c|c|c|c|c|c|c|c|}
\hline $\mathcal{N}_{h}$ & $h$ & $e_{1}\left(u_{1}\right)$ & $r_{1}\left(u_{1}\right)$ & $e_{0}\left(u_{1}\right)$ & $r_{0}\left(u_{1}\right)$ & $e_{1}\left(u_{2}\right)$ & $r_{1}\left(u_{2}\right)$ & $e_{0}\left(u_{2}\right)$ & $r_{0}\left(u_{2}\right)$ \\
\hline 9 & 3.7922 & $1.0417 \mathrm{e}-1$ & - & $7.7254 \mathrm{e}-1$ & - & $8.6421 \mathrm{e}-2$ & - & $3.7412 \mathrm{e}-1$ & \\
\hline 27 & 2.0847 & 7.6978e-2 & 0.5042 & $1.3049 \mathrm{e}-1$ & 2.9640 & $4.7942 \mathrm{e}-2$ & 0.9821 & $4.2062 \mathrm{e}-2$ & 3.6424 \\
\hline 96 & 0.9922 & $4.1841 \mathrm{e}-2$ & 0.8212 & $2.9158 \mathrm{e}-2$ & 2.0185 & $2.2313 \mathrm{e}-2$ & 1.0302 & $8.2372 \mathrm{e}-3$ & 2.1962 \\
\hline 333 & 0.4961 & $2.1628 \mathrm{e}-2$ & 0.9548 & $9.0312 \mathrm{e}-3$ & 1.7958 & $1.0757 \mathrm{e}-2$ & 1.0557 & $2.1504 \mathrm{e}-3$ & 1.9432 \\
\hline 1265 & 0.2549 & $1.0694 \mathrm{e}-2$ & 1.0524 & $2.3387 \mathrm{e}-3$ & 2.0188 & $5.2402 \mathrm{e}-3$ & 1.0746 & $4.8176 \mathrm{e}-4$ & 2.2353 \\
\hline 4972 & 0.1312 & $5.3102 \mathrm{e}-3$ & 1.0452 & $6.1799 e-4$ & 1.9870 & $2.6244 \mathrm{e}-3$ & 1.0324 & $8.4301 \mathrm{e}-5$ & 2.6023 \\
\hline 19732 & 0.0664 & $2.6260 \mathrm{e}-3$ & 1.0481 & $1.4542 \mathrm{e}-4$ & 2.1536 & $1.3102 \mathrm{e}-3$ & 1.0340 & $3.0172 \mathrm{e}-5$ & 1.5294 \\
\hline
\end{tabular}
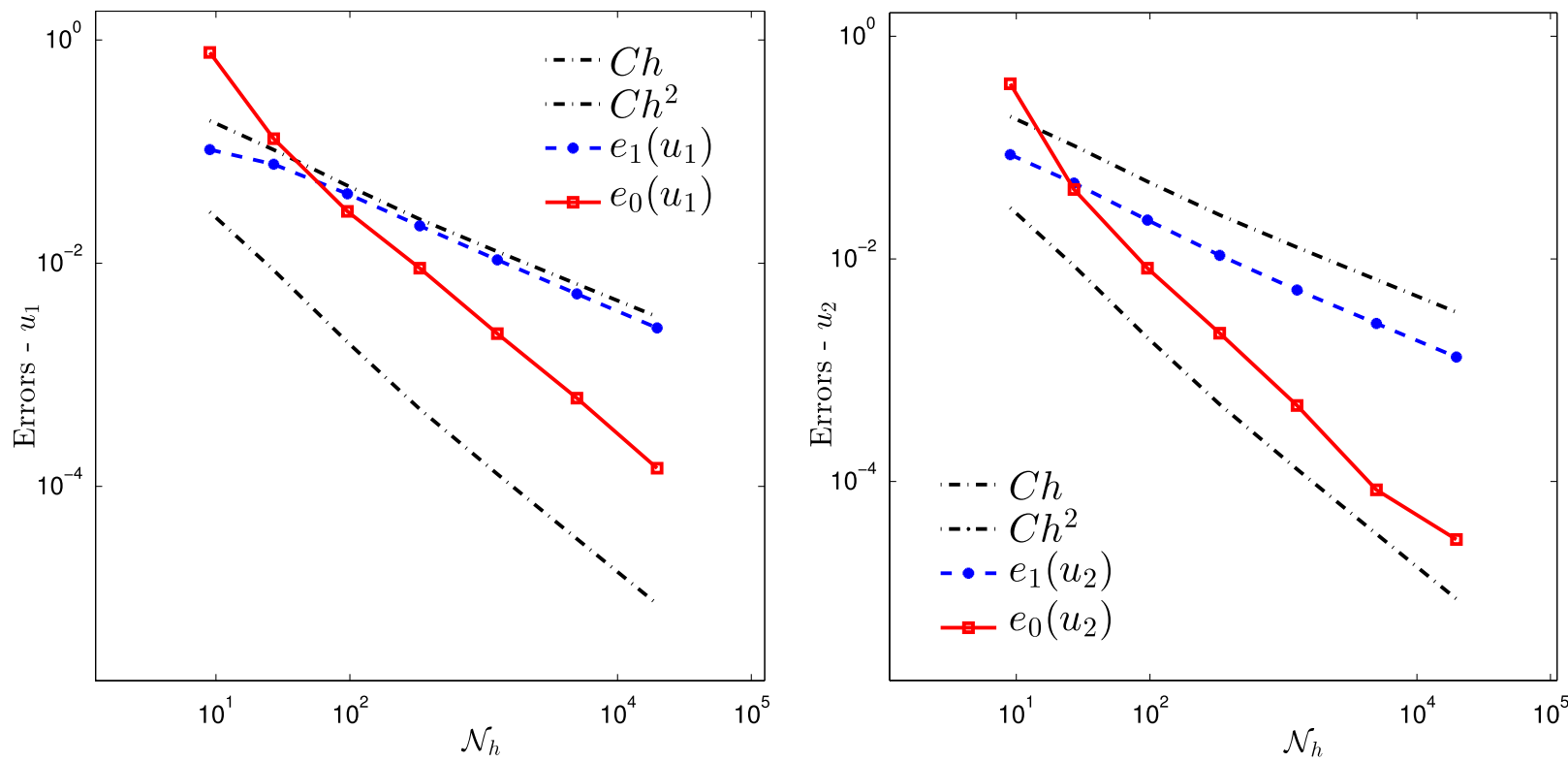

Fig. 4. Validation test: Convergence histories for the finite volume element method applied to (4.1). The displayed quantities correspond to relative errors and convergence rates in the $L^{2}$-norm and $H^{1}$-seminorm for species $u_{1}$ (left) and $u_{2}$ (right).

an additive Schwarz domain decomposition strategy [38]. Basically, the global preconditioner is defined by the expression $P^{-1}=\sum_{k=1}^{M} R^{T} P_{i}^{-1} R$, where $P_{k}$ is the local preconditioner associated to the processor $k$ (typically a local KLU factorization), $M$ stands for the total number of processors and $R$ is the usual restriction operator to extract part of the global matrix. We employ one level of overlap among processors.

\subsection{Example 1: Mixed patterns on a square and on a cube}

We start by studying the behavior of problem (1.1) at the bifurcation point corresponding to $D_{12}=22.2665$. According to (2.6), we obtain the critical wavenumber $\left|\mathbf{k}_{c}\right|=3.1741$ and we expect patterns to appear. The time evolution of the system is performed on the square $\Omega=(0, L)^{2}$ for $t=1,10,25$. The unstructured primal mesh contains 15364 triangles and 7683 nodes and a (heuristically determined) timestep of $\Delta t=1 e-3$ is employed. The same test is done on the cube $\Omega=(0, L)^{3}$, where the computational mesh consists of 43859 vertices and 241984 tetrahedra. In Fig. 6 we depict the approximate solutions for both tests, where hexagon-stripe mixtures are displayed. Here stripes and spots coexist both in the square and in the cube.

\subsection{Example 2: Spotted patterns on a square and on a cube}

In this example we observe the behavior above the threshold and we choose $D_{12}=24$. For this parameter we obtain the critical wavenumber $\left|\mathbf{k}_{c}\right|=3.7249$. In Fig. 7 we show the evolution of the spatial patterns on a square $\Omega=(0, L)^{2}$, also for times $t=1,10,25$, along with the computations performed on the cube $\Omega=(0, L)^{3}$. Space and time discretizations coincide with those from Example 1. From Fig. 7 we observe spotted patterns both in the square and in the cube. 

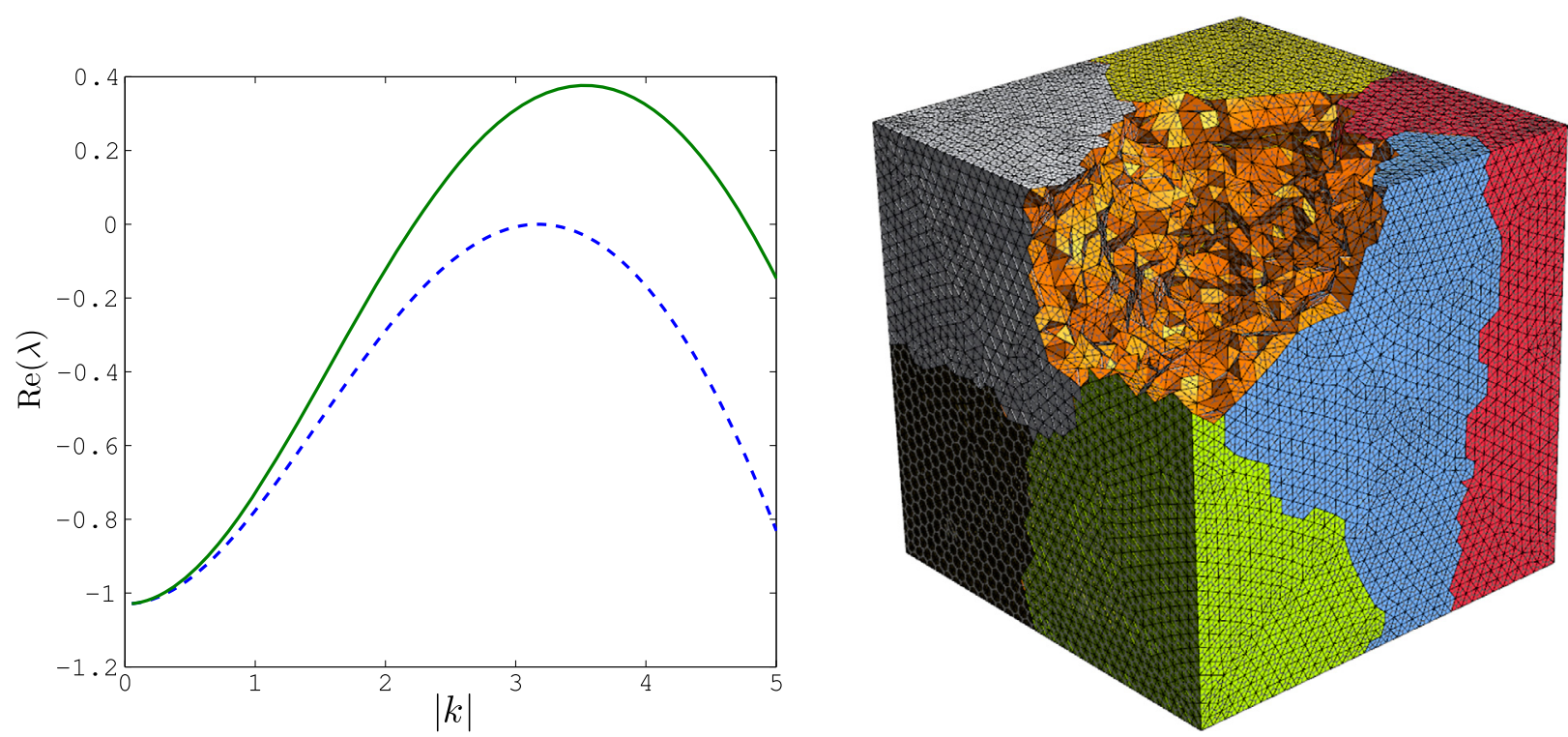

Fig. 5. Left: Examples of the dispersion relation of (1.1) for $D_{12}=22.2665$ (dashed blue), and $D_{12}=24$ (green line). Right: Partition of the actual primal and dual meshes into 16 non-overlapping subdomains, each color representing a different subdomain. (For interpretation of the references to color in this figure legend, the reader is referred to the web version of this article.)

Table 2

Example 3: Study of the influence of diffusion coefficients in the stability of the method, measured by the GMRES iteration count (to reach the desired tolerance), as the meshsize decreases.

\begin{tabular}{rllll}
\hline $\mathcal{N}_{h}$ & $D_{12} / D_{21}=3$ & $D_{12} / D_{21}=44.533$ & $D_{21} / D_{12}=48$ & $D_{21} / D_{12}=64$ \\
\hline 2384 & 5.84 & 6.21 & 6.40 & 7.24 \\
5177 & 5.99 & 6.75 & 6.58 & 7.18 \\
9636 & 7.10 & 7.04 & 7.09 & 7.85 \\
18671 & 6.82 & 7.85 & 7.15 & 7.98 \\
43859 & 7.42 & 6.79 & 6.91 & 7.25 \\
96378 & 6.92 & 6.34 & 7.33 & 8.78 \\
\hline
\end{tabular}

\subsection{Example 3: Effect of increased cross-diffusion and scalability test}

We increase further the cross-diffusion coefficient $D_{12}=32$ and run the same tests as in the previous examples. The results are collected in Fig. 8. We observe a larger amplitude of the patterns (higher gradients) for both species. The exhibited patterns are of labyrinthine type in both the square and the cube.

Next we perform a basic sensitivity analysis for the cross-diffusion coefficients. We study the behavior of the average iteration count for different cross-diffusion ratios as the meshsize decreases (the remaining coefficients are kept constant). A fixed fine timestep is used in all cases and we simulate the process up to $t=10$. From Table 2 it is straightforward to see that, for cross-diffusion regimes as those analyzed here, the method remains stable (in the sense that the number of iterations remains approximately constant).

In addition we report on the weak scaling of our reaction-diffusion solver, where the problem size and the multiprocessor count are simultaneously expanded. Ideally, the goal is to achieve constant (or, in practice, slightly increasing) time-to-solution for larger problems. For this example the cross-diffusion is $D_{12}=32$ and only 100 time steps are used in each run of the simulation performed on the cube, where we progressively increase the number of processors and refine the mesh, so that the ratio \#dof/\#processors is kept approximately constant. The results in Table 3 show the performance of the solver measured in terms of GMRES iterations to convergence of the linear solver, time per GMRES solution and CPU time per timestep. We conclude that the chosen preconditioner scales reasonably up to 192 processors.

\subsection{Example 4: Pattern selection on a disk and on a cube}

We study the influence of the cross-diffusion on a smaller domain of different shape. A series of simulations are performed on a disk of diameter $L=20$, where the primal mesh consists of 4710 elements and 2356 vertices. The Brusselator system is evolved until the solution reaches inhomogeneous stationary states and we depict in Fig. 9 the results for a varying cross-diffusion coefficient (only the $u_{1}$-field is presented). Here is more clearly seen the change of patterns according to the 

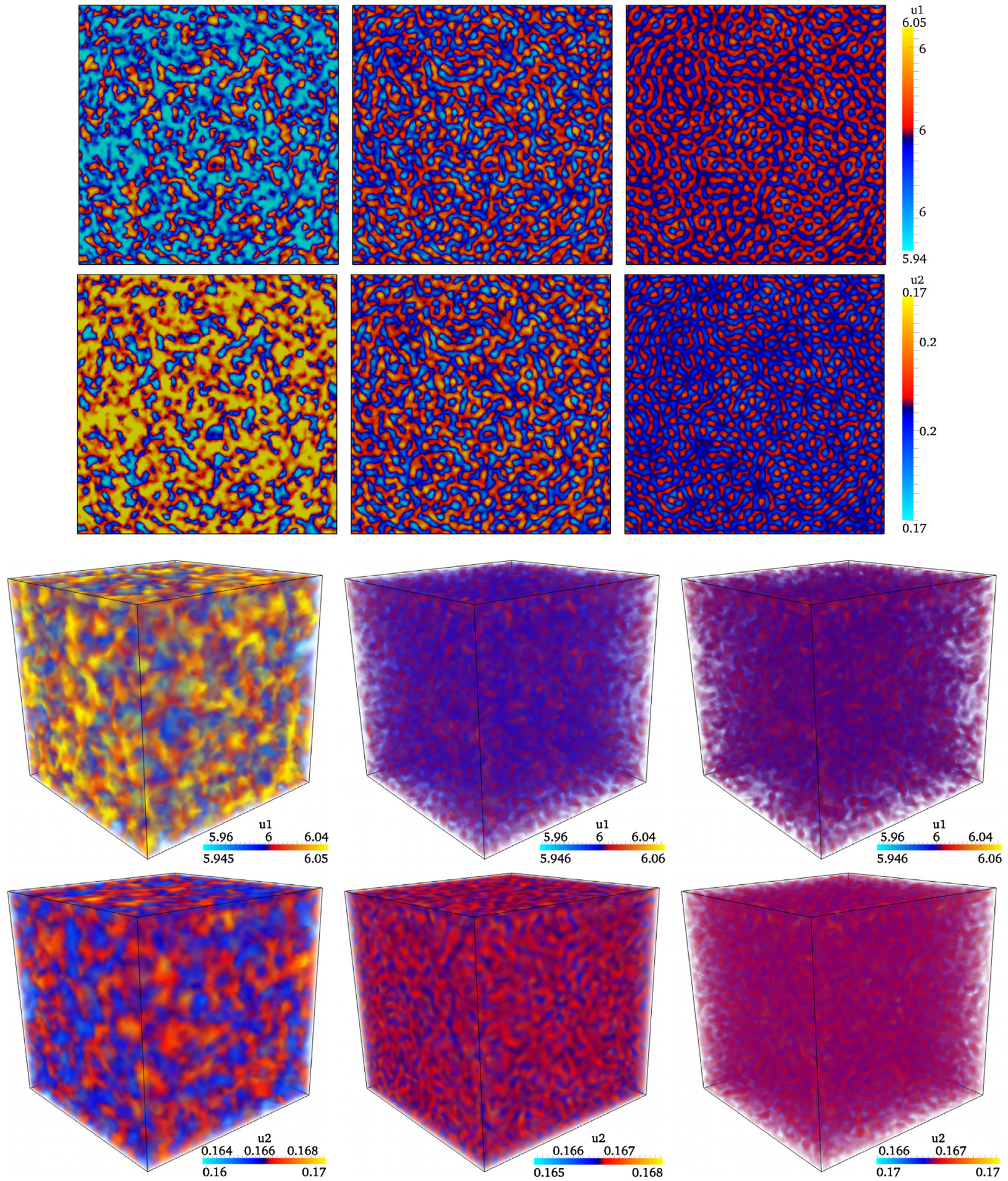

Fig. 6. Example 1: Snapshots of the numerical approximation ( $u_{1}$ top, $u_{2}$ bottom) of the Brusselator system with cross-diffusion $D_{12}=22.2665$. Simulations performed on a square and on a cube for times $t=1,10,25$ (left, middle, right).

cross-diffusion effect. With $D_{12}=20$ we observe that the initial perturbation of the equilibrium vanishes and the solution reaches the homogeneous equilibrium, whereas the increase of $D_{12}$ over the threshold yields the formation of spotted and labyrinthine patterns. The same behavior is evidenced in Fig. 10, where we show the approximate steady state solutions on a smaller cube $\Omega=(0,20)^{3}$. 

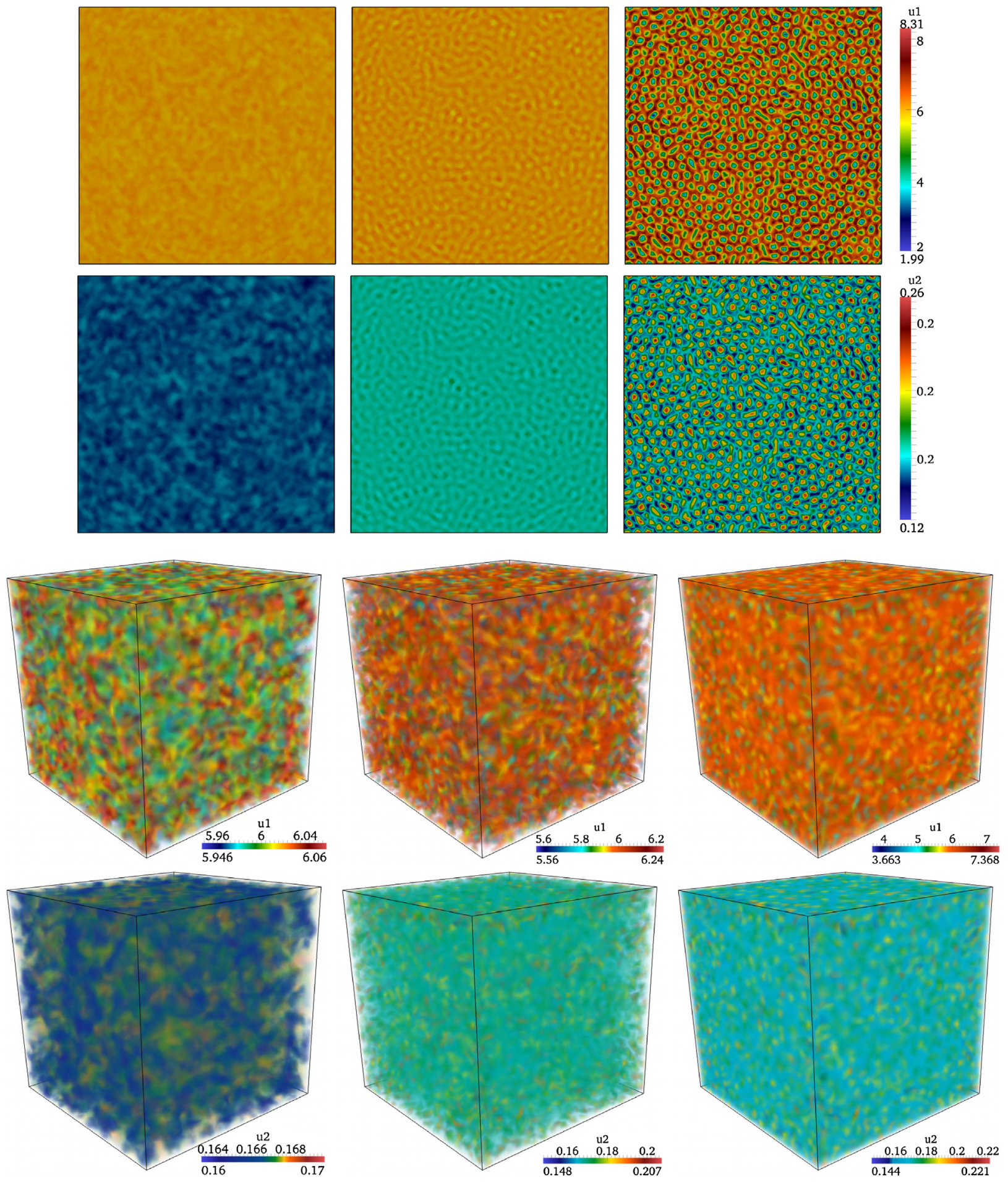

Fig. 7. Example 2: Snapshots of the numerical approximation ( $u_{1}$ top, $u_{2}$ bottom) of the Brusselator system with cross-diffusion $D_{12}=24$. Simulations performed on a square and on a cube for times $t=1,10,25$ (left, middle, right).

\section{Concluding remarks}

In this paper we proposed a finite volume element method for a reaction-cross-diffusion system modeling an autocatalytic chemical reaction. The stability of the Brusselator system has been addressed in detail. Experimental convergence rates were obtained for the FVE approximations and several numerical examples in two and three spatial dimensions verify 

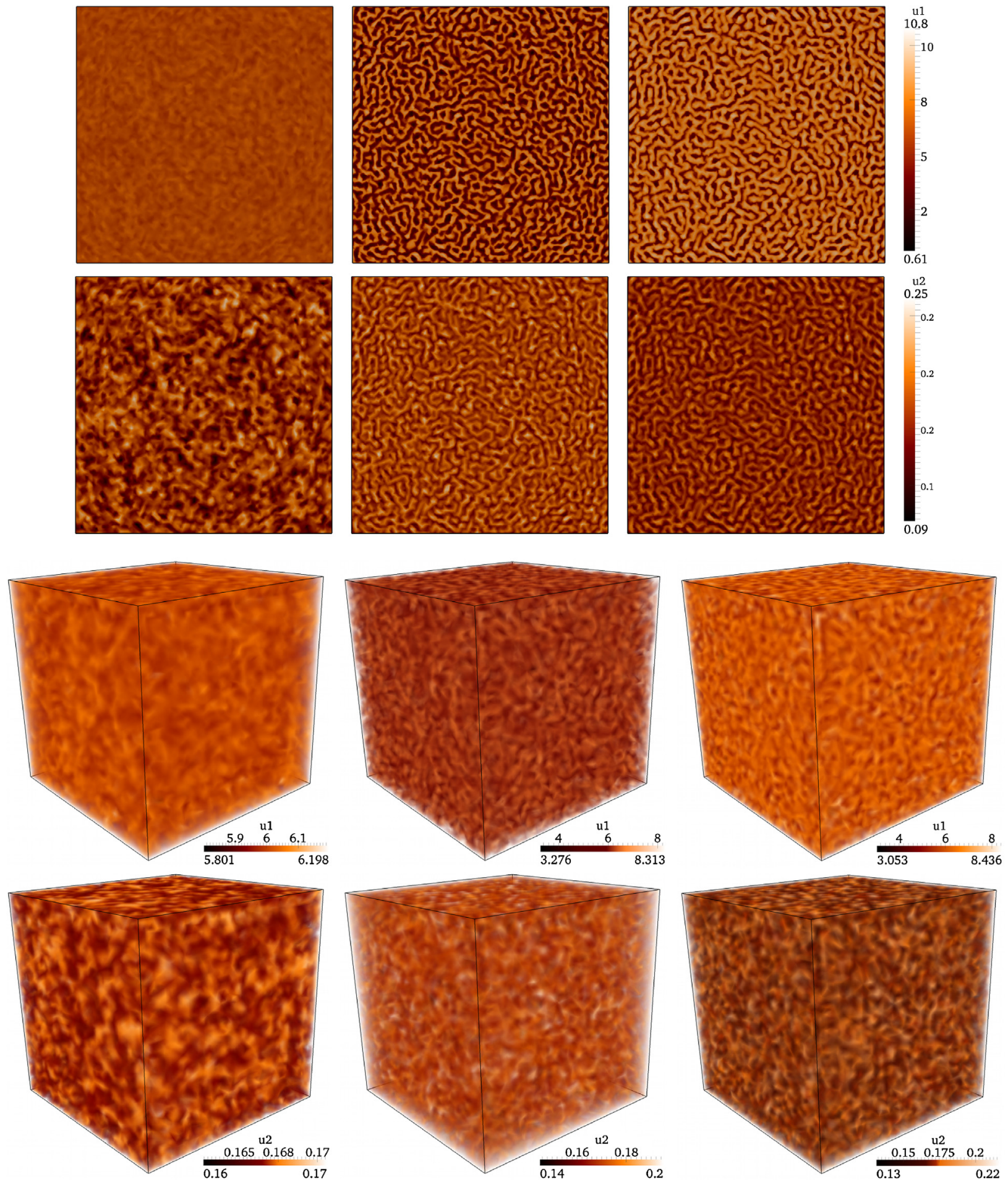

Fig. 8. Example 3: Snapshots of the numerical approximation $\left(u_{1}\right.$ top, $u_{2}$ bottom) of the Brusselator system with cross-diffusion $D_{12}=32$. Simulations performed on a square and on a cube for times $t=1,10,25$ (left, middle, right).

the expected results in terms of behavior of the generated patterns. We have shown that the cross-diffusion effect induces the formation of patterns via Turing mechanisms, also in the three-dimensional case.

The main limitation of the proposed method is the difficulty of deriving high order spatial approximations, and the coupling of conforming and nonconforming fields without resorting to complicated strategies as e.g. those in [11]. Other aspects that are postponed for a following study include the impact of high anisotropy of the medium and robustness 
Table 3

Example 3: Weak scalability of the reaction-diffusion solver, measured by the average iteration count and average CPU time.

\begin{tabular}{cllr}
\hline Processors & GMRES iterations & Time per GMRES solution & CPU time per timestep \\
\hline 24 & 6.52 & 35.21 & 81.77 \\
48 & 8.43 & 39.27 & 87.45 \\
96 & 8.71 & 40.19 & 113.02 \\
192 & 8.67 & 55.04 & 125.40 \\
\hline
\end{tabular}
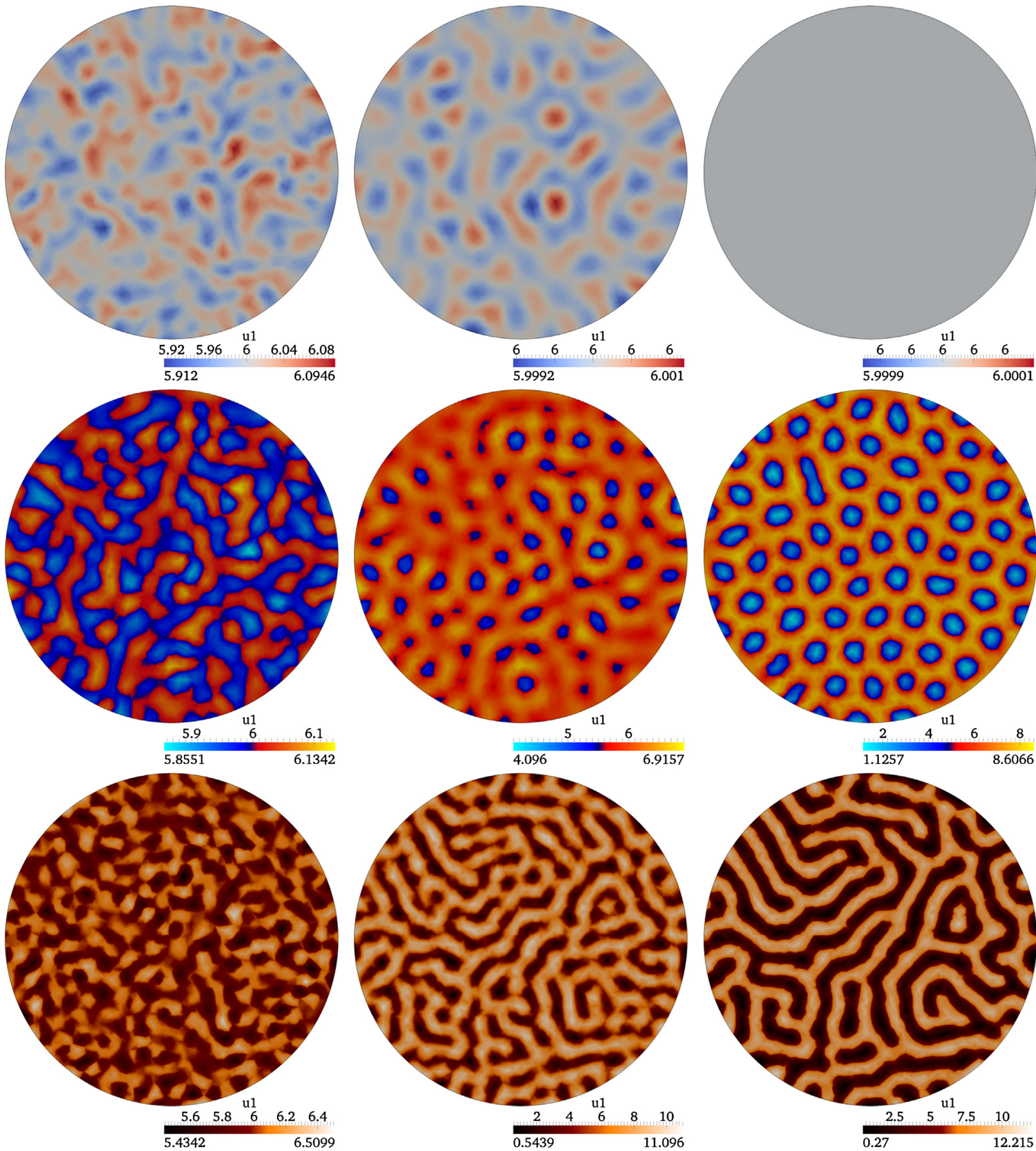

Fig. 9. Example 4: FVE approximation of species $u_{1}$ for cross-diffusion $D_{12}=22.2665,24,32$ (top, middle and bottom, respectively). Simulations performed on a disk for times $t=1,10,50$ (left, middle, right). 

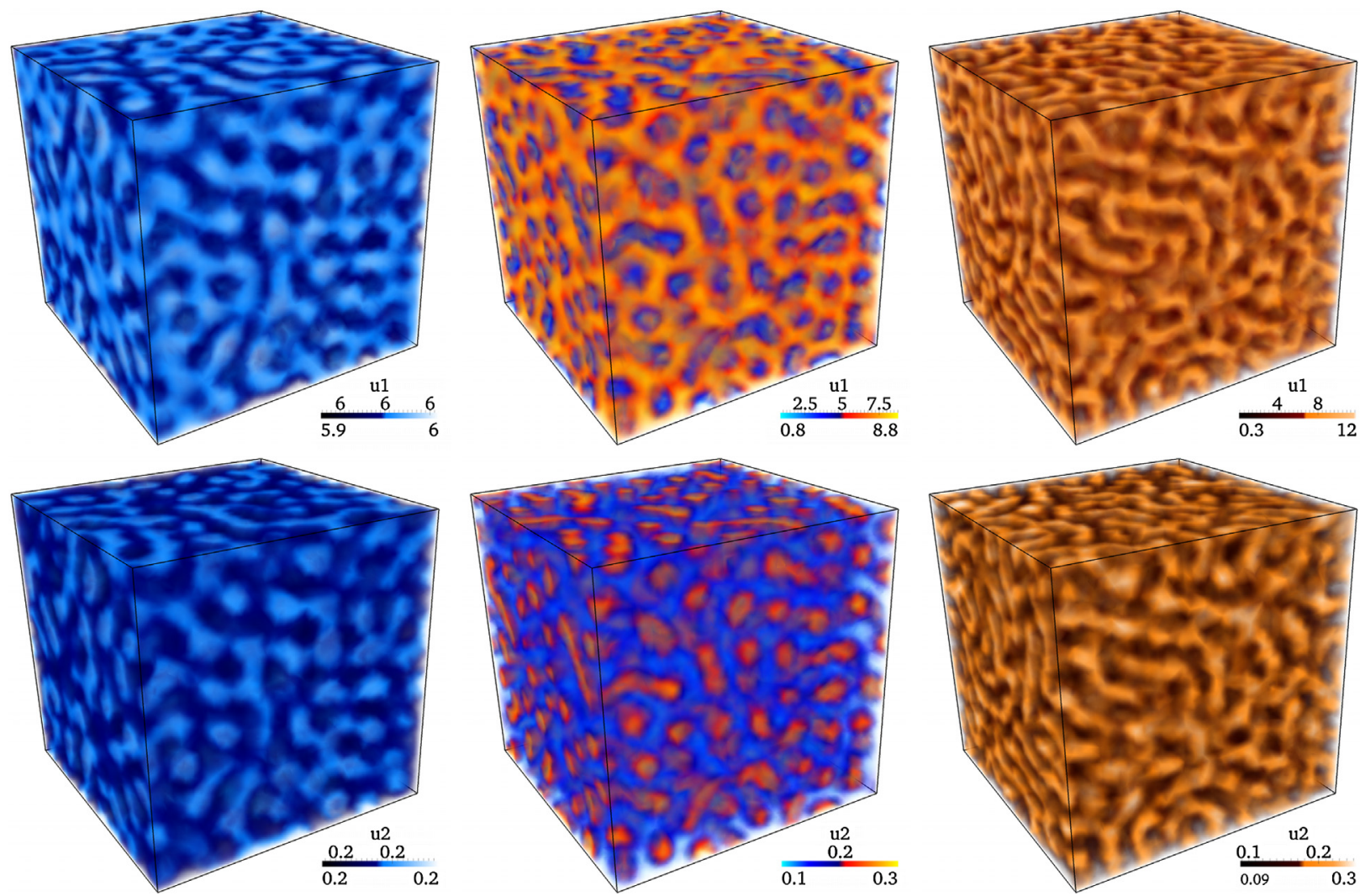

Fig. 10. Example 4: FVE approximation of species $u_{1}$ (top) and $u_{2}$ (bottom) for cross-diffusion values $D_{12}=22.2665,24,32$ (left, middle and right, respectively). Simulations performed on $\Omega=(0,20)^{3}$ until $T=50$.

of the method in presence of stretched primal grids (see e.g. [1]). In addition, rigorous comparison with experimental results is still a difficult task, however cross-diffusion effects could be introduced in laboratory experiments following the protocols described in [9]. Further extensions of our results (currently under development) include the study of pattern formation in growing domains (see e.g. [31]), and its relation with applications in biology (excitable media [10] and limb morphogenesis [50]).

\section{Acknowledgements}

R. Ruiz-Baier acknowledges support by the European Research Council Advanced Grant "Mathcard, Mathematical Modelling and Simulation of the Cardiovascular System", Project ERC-2008-AdG 227058, Z. Lin is partially supported by PRC grant NSFC-11071209 and NSFC-11371311, and C. Tian is partially supported by PRC grant NSFC-11201406 and Qinglan Project. The authors also thank Raimund Bürger for stimulating discussions on the subject of this paper.

\section{Appendix A. Proof of Theorem 2.1}

The linearization of (1.1) around the state $\mathbf{u}^{*}$ can be expressed by

$$
\frac{\partial \mathbf{u}}{\partial t}=\left(D \Delta+\mathbf{G}_{u}\left(\mathbf{u}^{*}\right)\right) \mathbf{u}
$$

where $D=\operatorname{diag}\left(D_{11}, D_{22}\right)$ because of $D_{12}=D_{21}=0$, and

$$
\mathbf{G}_{u}\left(\mathbf{u}^{*}\right)=\left(\begin{array}{cc}
\beta-1 & \alpha^{2} \\
-\beta & -\alpha^{2}
\end{array}\right) \text {. }
$$

We define the Banach space

$$
\mathbf{X}:=\left\{\mathbf{u} \in\left[C^{1}(\bar{\Omega})\right]^{2}: \frac{\partial u_{1}}{\partial \eta}=\frac{\partial u_{2}}{\partial \eta}=0 \text { on } \partial \Omega\right\} .
$$




$$
0=\mu_{1}<\mu_{2}<\cdots \rightarrow \infty,
$$

be the eigenvalues of the operator $-\Delta$ on $\Omega$ under homogeneous Neumann boundary conditions, and $E\left(\mu_{i}\right)$ be the space of eigenfunctions corresponding to $\mu_{i}$. If $\mathbf{X}_{i j}:=\left\{\mathbf{c} \cdot \phi_{i j}: \mathbf{c} \in \mathbb{R}^{2}\right\}$, where $\left\{\phi_{i j}\right\}$ are orthonormal basis of $E\left(\mu_{i}\right)$ for $j=1, \ldots, \operatorname{dim} E\left(\mu_{i}\right)$, then we have the following space decomposition

$$
\mathbf{x}=\bigoplus_{i=1}^{\infty} \mathbf{x}_{i}, \quad \text { where } \mathbf{x}_{i}=\bigoplus_{j=1}^{\operatorname{dim} E\left(\mu_{i}\right)} \mathbf{x}_{i j}
$$

Therefore the space $\mathbf{X}_{i}$ is invariant under the operator $D \Delta+\mathbf{G}_{u}\left(\mathbf{u}^{*}\right)$, and $\lambda$ is an eigenvalue of this operator on $\mathbf{X}_{i}$, if and only if it is an eigenvalue of the matrix $-\mu_{i} D+\mathbf{G}_{u}\left(\mathbf{u}^{*}\right)$.

A direct calculation shows that the characteristic polynomial of $-\mu_{i} D+\mathbf{G}_{u}\left(\mathbf{u}^{*}\right)$ is given by

$$
\psi_{i}(\lambda)=\lambda^{2}+B_{i} \lambda+C_{i}
$$

where

$$
B_{i}=\alpha^{2}+1-\beta+\left(D_{11}+D_{22}\right) \mu_{i}, \quad C_{i}=D_{11} D_{22} \mu_{i}^{2}+\left[\alpha^{2} D_{11}+(1-\beta) D_{22}\right] \mu_{i}+\alpha^{2} .
$$

It is straightforward to verify that $B_{i}$ and $C_{i}$ are positive if and only if (2.1) holds. Thus, for each $i \geqslant 1$, the two roots of $\psi_{i}(\lambda)=0$ all have negative real parts, and this concludes the proof.

\section{Appendix B. Proof of Theorem 2.2}

By

$$
\bar{u}_{1}=\frac{1}{|\Omega|} \int_{\Omega} u_{1}(x, t) d x
$$

we will denote the mean value of a function $u_{1}$ on $\Omega$ at time $t$. Let us consider the function

$$
J(t):=\int_{\Omega}\left(u_{1}(x, t)-\bar{u}_{1}\right)^{2} d x+\int_{\Omega}\left(u_{2}(x, t)-\bar{u}_{2}\right)^{2} d x=2\left(J_{1}(t)+J_{2}(t)\right),
$$

where $J_{i}(t)=\frac{1}{2} \int_{\Omega}\left(u_{i}(x, t)-\bar{u}_{i}\right)^{2} d x$ for $i=1,2$. Differentiation of $J_{1}$ with respect to $t$ yields

$$
\begin{aligned}
\frac{d}{d t} J_{1}(t) & =\int_{\Omega}\left(u_{1}-\bar{u}_{1}\right)\left(\frac{d u_{1}}{d t}-\frac{d \bar{u}_{1}}{d t}\right) d x \\
& =\int_{\Omega}\left(u_{1}-\bar{u}_{1}\right)\left(D_{11} \Delta u_{1}+D_{12} \Delta u_{2}+u_{1}^{2} u_{2}-(\beta+1) u_{1}-\bar{u}_{1}^{2} \bar{u}_{2}+(\beta+1) \bar{u}_{1}\right) d x \\
& =\int_{\Omega}\left(u_{1}-\bar{u}_{1}\right)\left(D_{11} \Delta u_{1}+D_{12} \Delta u_{2}\right) d x+\int_{\Omega}\left(u_{1}-\bar{u}_{1}\right)\left[\left(-\beta-1+\bar{u}_{2}\left(u_{1}+\bar{u}_{1}\right)\right)\left(u_{1}-\bar{u}_{1}\right)+u_{1}^{2}\left(u_{2}-\bar{u}_{2}\right)\right] .
\end{aligned}
$$

After using the homogeneous Neumann boundary conditions of (1.1), it follows that

$$
\begin{aligned}
\frac{d}{d t} J_{1}(t)= & -D_{11} \int_{\Omega}\left|\nabla u_{1}\right|^{2} d x-D_{12} \int_{\Omega} \nabla u_{1} \cdot \nabla u_{2} d x \\
& +\int_{\Omega}\left(u_{1}-\bar{u}_{1}\right)\left[\left(-\beta-1+\bar{u}_{2}\left(u_{1}+\bar{u}_{1}\right)\right)\left(u_{1}-\bar{u}_{1}\right)+u_{1}^{2}\left(u_{2}-\bar{u}_{2}\right)\right] .
\end{aligned}
$$

In a similar way,

$$
\begin{aligned}
\frac{d}{d t} J_{2}(t) & =\int_{\Omega}\left(u_{2}-\bar{u}_{2}\right)\left(\frac{d u_{2}}{d t}-\frac{d \bar{u}_{2}}{d t}\right) d x \\
& =\int_{\Omega}\left(u_{2}-\bar{u}_{2}\right)\left(D_{21} \Delta u_{1}+D_{22} \Delta u_{2}+\beta u_{1}-u_{1}^{2} u_{2}-\beta \bar{u}_{1}+\bar{u}_{1}^{2} \bar{u}_{2}\right) d x
\end{aligned}
$$




$$
\begin{aligned}
= & -D_{21} \int_{\Omega} \nabla u_{1} \cdot \nabla u_{2} d x-D_{22} \int_{\Omega}\left|\nabla u_{1}\right|^{2} d x \\
& +\int_{\Omega}\left(u_{2}-\bar{u}_{2}\right)\left[\left(\beta-u_{2}\left(u_{1}+\bar{u}_{1}\right)\right)\left(u_{1}-\bar{u}_{1}\right)-\bar{u}_{1}^{2}\left(u_{2}-\bar{u}_{2}\right)\right] .
\end{aligned}
$$

Combining (B.1) and (B.2), we have

$$
\begin{aligned}
\frac{d\left(J_{1}+J_{2}\right)}{d t}= & -D_{11} \int_{\Omega}\left|\nabla u_{1}\right|^{2} d x-\left(D_{12}+D_{21}\right) \int_{\Omega} \nabla u_{1} \cdot \nabla u_{2} d x-D_{22} \int_{\Omega}\left|\nabla u_{2}\right|^{2} d x \\
& +\int_{\Omega}\left(u_{1}-\bar{u}_{1}\right)^{2}\left[-\beta-1+\bar{u}_{2}\left(u_{1}+\bar{u}_{1}\right)\right] d x+\int_{\Omega}\left(u_{2}-\bar{u}_{2}\right)^{2}\left[-\bar{u}_{1}^{2}\right] d x \\
& +\int_{\Omega}\left(u_{1}-\bar{u}_{1}\right)\left(u_{2}-\bar{u}_{2}\right)\left[\beta+u_{1}^{2}-u_{2}\left(u_{1}+\bar{u}_{1}\right)\right] d x .
\end{aligned}
$$

Since $0 \leqslant u_{1}(x, t) \leqslant K_{1}, 0 \leqslant u_{2}(x, t) \leqslant K_{2}$ for all $(x, t) \in \Omega_{T}$, we have

$$
\begin{aligned}
\frac{d\left(J_{1}+J_{2}\right)}{d t} \leqslant & -D_{11} \int_{\Omega}\left|\nabla u_{1}\right|^{2} d x+\left(D_{12}+D_{21}\right) \int_{\Omega}\left|\nabla u_{1} \cdot \nabla u_{2}\right| d x-D_{22} \int_{\Omega}\left|\nabla u_{2}\right|^{2} d x \\
& +\left(-\beta-1+2 K_{1} K_{2}\right) \int_{\Omega}\left(u_{1}-\bar{u}_{1}\right)^{2} d x+\left(\beta+K_{1}^{2}+2 K_{1} K_{2}\right) \int_{\Omega}\left|\left(u_{1}-\bar{u}_{1}\right)\left(u_{2}-\bar{u}_{2}\right)\right| d x .
\end{aligned}
$$

Applying Hölder's and Poincaré inequalities yields

$$
\begin{aligned}
\frac{d\left(J_{1}+J_{2}\right)}{d t} \leqslant & -D_{11} \int_{\Omega}\left|\nabla u_{1}\right|^{2} d x+\left(D_{12}+D_{21}\right)\left(\int_{\Omega}\left|\nabla u_{1}\right|^{2} d x\right)^{1 / 2}\left(\int_{\Omega}\left|\nabla u_{2}\right|^{2} d x\right)^{1 / 2} \\
& -D_{22} \int_{\Omega}\left|\nabla u_{2}\right|^{2} d x+\frac{-\beta-1+2 K_{1} K_{2}}{\mu_{2}} \int_{\Omega}\left|\nabla u_{1}\right|^{2} d x \\
& +\frac{\beta+K_{1}^{2}+2 K_{1} K_{2}}{\mu_{2}}\left(\int_{\Omega}\left|\nabla u_{1}\right|^{2} d x\right)^{1 / 2}\left(\int_{\Omega}\left|\nabla u_{2}\right|^{2} d x\right)^{1 / 2} \\
= & \left.-D_{11}+\frac{-\beta-1+2 K_{1} K_{2}}{\mu_{2}}\right) \int_{\Omega}\left|\nabla u_{1}\right|^{2} d x-D_{22} \int_{\Omega}\left|\nabla u_{2}\right|^{2} d x \\
& +\left(D_{12}+D_{21}+\frac{\beta+K_{1}^{2}+2 K_{1} K_{2}}{\mu_{2}}\right)\left(\int_{\Omega}\left|\nabla u_{1}\right|^{2} d x\right)^{1 / 2}\left(\int_{\Omega}\left|\nabla u_{2}\right|^{2} d x\right)^{1 / 2} .
\end{aligned}
$$

Since (2.2) holds, it follows from Cauchy's inequality, that

$$
\frac{d\left(J_{1}+J_{2}\right)}{d t}<0
$$

Therefore, $J(t) \rightarrow 0$ exponentially as $t \rightarrow 0$, which implies that problem (1.1) does not possess an inhomogeneous steady state.

\section{Appendix C. Proof of Theorem 2.3}

Let

$$
D=\left(\begin{array}{ll}
D_{11} & D_{12} \\
D_{21} & D_{22}
\end{array}\right)
$$

denote the diffusion matrix. Direct computation gives that the characteristic polynomial of $-\mu_{i} D+\mathbf{G}_{u}\left(\mathbf{u}^{*}\right)$ is

$$
\psi_{i}(\lambda)=\lambda^{2}+\bar{B}_{i} \lambda+\bar{C}_{i}
$$

where 


$$
\begin{aligned}
& \bar{B}_{i}=\alpha^{2}+1-\beta+\left(D_{11}+D_{22}\right) \mu_{i}, \\
& \bar{C}_{i}=\left(D_{11} D_{22}-D_{12} D_{21}\right) \mu_{i}^{2}+\left[\alpha^{2} D_{11}+(1-\beta) D_{22}-\beta D_{12}+\alpha^{2} D_{21}\right] \mu_{i}+\alpha^{2} .
\end{aligned}
$$

Let $\lambda_{1}\left(\mu_{i}\right)$ and $\lambda_{2}\left(\mu_{i}\right)$ be the two roots of $\psi_{i}(\lambda)=0$. We then have

$$
\lambda_{1}\left(\mu_{i}\right)+\lambda_{2}\left(\mu_{i}\right)=\bar{B}_{i} \text { and } \lambda_{1}\left(\mu_{i}\right) \lambda_{2}\left(\mu_{i}\right)=\bar{C}_{i} .
$$

In order to ensure that $\operatorname{Re} \lambda_{1}\left(\mu_{i}\right)<0$ and $\operatorname{Re} \lambda_{2}\left(\mu_{i}\right)>0$, a necessary and sufficient condition is $\bar{C}_{i}<0$ (based on the fact that $\left.\bar{B}_{i}>0\right)$.

Next, we look for conditions equivalent to $\bar{C}_{i}<0$. Notice that $\bar{C}_{i}$ is a quadratic polynomial with respect to $\mu_{i}$. In addition, there are two values of $D_{11} D_{22}-D_{12} D_{21}$ to be considered.

Case (i) $D_{11} D_{22}-D_{12} D_{21}>0$; If and only if

$$
\frac{1}{\beta}\left[\alpha^{2}\left(D_{11}+D_{21}\right)+(1-\beta) D_{22}+2 \alpha \sqrt{D_{11} D_{22}-D_{12} D_{21}}\right] \leqslant D_{12},
$$

there exists a positive constant

$$
\mu_{i}=\frac{-\alpha^{2} D_{11}-(1-\beta) D_{22}+\beta D_{12}-\alpha^{2} D_{21}}{2\left(D_{11} D_{22}-D_{12} D_{21}\right)},
$$

such that $\bar{C}_{i}<0$.

Case (ii) $D_{11} D_{22}-D_{12} D_{21} \leqslant 0$; We set

$$
\mu_{i}=\frac{\alpha^{2} D_{11}+(1-\beta) D_{22}-\beta D_{12}+\alpha^{2} D_{21}}{2\left(D_{11} D_{22}-D_{12} D_{21}\right)},
$$

and then $\bar{C}_{i}<0$.

The above argument shows that (2.3) holds if and only if Turing patterns arise, and this completes the proof.

\section{References}

[1] M. Afif, B. Amaziane, G. Kunert, Z. Mghazli, S. Nicaise, A posteriori error estimation of a finite volume discretization on anisotropic meshes, J. Sci. Comput. 43 (2010) 183-200.

[2] B. Andreianov, M. Bendahmane, R. Ruiz-Baier, Analysis of a finite volume method for a cross-diffusion model in population dynamics, Math. Models Methods Appl. Sci. 21 (2011) 307-344.

[3] R.E. Bank, D.J. Rose, Some error estimates for the box method, SIAM J. Numer. Anal. 24 (1987) 777-787.

[4] T. Bansagi, V.K. Vanag, I.R. Epstein, Tomography of reaction-diffusion microemulsions reveals three-dimensional turing patterns, Science 331 (2011) 1309-1312.

[5] J.W. Barrett, J.F. Blowey, Finite element approximation of a nonlinear cross-diffusion population model, Numer. Math. 98 (2004) $195-221$.

[6] R.A. Barrio, C. Varea, J.L. Aragón, A two-dimensional numerical study of spatial pattern formation in interacting Turing systems, Bull. Math. Biol. 61 (1999) 483-505.

[7] M. Bendahmane, R. Bürger, R. Ruiz-Baier, K. Schneider, Adaptive multiresolution schemes with local time stepping for two-dimensional degenerate reaction-diffusion systems, Appl. Numer. Math. 59 (2009) 1668-1692.

[8] S. Berres, R. Ruiz-Baier, A fully adaptive numerical approximation for a two-dimensional epidemic model with nonlinear cross-diffusion, Nonlinear Anal., Real World Appl. 12 (2011) 2888-2903.

[9] J. Boissonade, Stationary structure induced along a reaction-diffusion front by a Turing symmetry breaking instability, J. Phys. France 49 (1988) $541-546$.

[10] R. Bürger, R. Ruiz-Baier, K. Schneider, Adaptive multiresolution methods for the simulation of waves in excitable media, J. Sci. Comput. 43 (2010) $261-290$.

[11] R. Bürger, R. Ruiz-Baier, H. Torres, A stabilized finite volume element formulation for sedimentation-consolidation processes, SIAM J. Sci. Comput. 34 (2012) B265-B289.

[12] V. Castets, E. Dulos, J. Boissonade, P. De Kepper, Experimental evidence of a sustained standing Turing-type nonequilibrium chemical pattern, Phys. Rev. Lett. 64 (1990) 2953-2956.

[13] L. Chen, A. Jüngel, Analysis of a multi-dimensional parabolic population model with strong cross-diffusion, SIAM J. Math. Anal. 36 (2004) $301-322$.

[14] E.J. Crampin, W.W. Hackborn, P.K. Maini, Pattern formation in reaction-diffusion models with nonuniform domain growth, Bull. Math. Biol. 64 (2002) $746-769$.

[15] P. De Kepper, I.R. Epstein, K. Kustin, M. Orban, Systematic design of chemical oscillators. Part 8. Batch oscillations and spatial wave patterns in chlorite oscillating systems, J. Phys. Chem. 86 (1982) 170-171.

[16] S. Descombes, M. Massot, Operator splitting for nonlinear reaction-diffusion systems with an entropic structure: Singular perturbation and order reduction, Numer. Math. 97 (2004) 667-698.

[17] R.I. Fernandes, G. Fairweather, An ADI extrapolated Crank-Nicolson orthogonal spline collocation method for nonlinear reaction-diffusion systems, J. Comput. Phys. 231 (2012) 6248-6267.

[18] G. Gambino, M.C. Lombardo, M. Sammartino, Pattern formation driven by cross-diffusion in a 2D domain, Nonlinear Anal.: Real World Appl. 14 (3) (2013) 1755-1779, http://dx.doi.org/10.1016/j.nonrwa.2012.11.009.

[19] A. Gierer, H. Meinhardt, A theory of biological pattern formation, Kybernetika 12 (1972) 30-39.

[20] V. Girault, A combined finite element and marker and cell method for solving Navier-Stokes equations, Numer. Math. 26 (1976) 39-59.

[21] A.A. Golovin, B.J. Matkowsky, V.A. Volpert, Turing pattern formation in the Brusselator model with superdiffusion, SIAM J. Appl. Math. 69 (2008 $251-272$. 
[22] P. Gray, S.K. Scott, Autocatalytic reactions in the isothermal, continuous stirred tank reactor: Oscillations and the instabilities in the system $A+2 B \rightarrow$ $3 B, B \rightarrow X$, Chem. Eng. Sci. 39 (1984) 1087-1097.

[23] E. Hairer, G. Wanner, Solving ordinary differential equations II, in: Springer Ser. Comput. Math., New York, 2002.

[24] D. Hilhorst, M. Vohralík, A posteriori error estimates for combined finite volume-finite element discretizations of reactive transport equations on nonmatching grids, Comput. Methods Appl. Mech. Eng. 200 (2011) 597-613.

[25] S. Hollis, R. Martin, M. Pierre, Global existence and boundedness in reaction-diffusion systems, SIAM J. Math. Anal. 18 (1987) 744-761.

[26] G. Karypis, V. Kumar, A parallel algorithm for multilevel graph partitioning and sparse matrix ordering, J. Parallel Distrib. Comput. 48 (1998) 71-85.

[27] R.D. Lazarov, S.Z. Tomov, Adaptive finite volume element method for convection-diffusion-reaction problems in 3-D, in: P. Minev, Y. Wong, Y. Lin (Eds.), Scientific Computing and Application, in: Adv. Comput. Theory Pract., vol. 7, Nova Science Publishing House, 2001, pp. 91-106.

[28] T. Leppänen, M. Karttunen, K. Kaski, R.A. Barrio, L. Zhang, A new dimension to Turing patterns, Physica D 168 (2002) $35-44$.

[29] J. Li, Z. Chen, W. Wu, Generalized Difference Methods for Differential Equations: Numerical Analysis of Finite Volume Methods, Marcel Dekker, New York, 2000.

[30] W.C. Lo, L. Chen, M. Wang, Q. Nie, A robust and efficient method for steady state patterns in reaction-diffusion systems, J. Comput. Phys. 231 (2012) 5062-5077.

[31] A. Madzvamuse, P.K. Maini, A.J. Wathen, A moving grid finite element method for the simulation of pattern generation by Turing models on growing domains, J. Sci. Comput. 24 (2005) 247-262.

[32] R.C. Mittal, R. Jiwari, Numerical solution of two-dimensional reaction-diffusion Brusselator system, Appl. Math. Comput. 217 (2011) 5404-5415.

[33] J.D. Murray, Mathematical Biology II: Spatial Models and Biomedical Applications, third edition, Springer-Verlag, New York, 2003.

[34] R. Nicolaides, The covolume approach to computing incompressible flows, in: M. Gunzburger, R. Nicolaides (Eds.), Incompressible Computational Fluid Dynamics, Cambridge University Press, Cambridge, UK, 1993, pp. 295-333.

[35] Q. Ouyang, H.L. Swinney, Transition from a uniform state to hexagonal and striped Turing patterns, Nature 352 (1991) 610-612.

[36] I. Prigogine, R. Lefever, Symmetry breaking instabilities in dissipative systems. II, J. Chem. Phys. 48 (1968) 1695-1700.

[37] A. Quarteroni, R. Ruiz-Baier, Analysis of a finite volume element method for the Stokes problem, Numer. Math. 118 (2011) 737-764.

[38] A. Quarteroni, A. Valli, Domain Decomposition Methods for Partial Differential Equations, Oxford University Press, UK, 1999.

[39] R. Ruiz-Baier, C. Tian, Mathematical analysis and numerical simulation of pattern formation under cross-diffusion, Nonlinear Anal., Real World Appl. 14 (2013) 601-612.

[40] Y. Saad, Iterative Methods for Sparse Linear Systems, PWS Publishing Co., 1996.

[41] J. Schnakenberg, Simple chemical reaction systems with limit cycle behavior, J. Theor. Biol. 81 (1979) 389-400.

[42] Siraj-ul-Islam, A. Ali, S. Haq, A computational modeling of the behavior of the two-dimensional reaction-diffusion Brusselator system, Appl. Math. Models 34 (2010) 3896-3909.

[43] C. Tian, L. Zhang, Z. Ling, The stability of a diffusion model of plankton allelopathy with spatio-temporal delays, Nonlinear Anal., Real World Appl. 10 (2009) 2036-2046.

[44] C. Tian, Z. Lin, Asymptotic behavior of solutions of a periodic diffusion system of plankton allelopathy, Nonlinear Anal., Real World Appl. 11 (2010) $1581-1588$.

[45] C. Tian, Coexistence and asymptotic periodicity in a competition model of plankton allelopathy, Acta Appl. Math. 113 (2011) 195-206.

[46] A. Turing, The chemical basis of morphogenesis, Philos. Trans. R. Soc. B 237 (1952) 37-72.

[47] V.K. Vanag, I.R. Epstein, Cross-diffusion and pattern formation in reaction-diffusion systems, Phys. Chem. Chem. Phys. 11 (2009) 897-912.

[48] J.G. Verwer, W.H. Hundsdorfer, B.P. Sommeijer, Convergence properties of the Runge-Kutta-Chebyshev method, Numer. Math. 57 (1990) 157-178.

[49] J.Y. Wakano, C. Hauert, Pattern formation and chaos in spatial ecological public goods games, J. Theor. Biol. 268 (2011) $30-38$.

[50] J. Zhu, Y.T. Zhang, S.A. Newman, M. Alber, Application of discontinuous Galerkin methods for reaction-diffusion systems in developmental biology, J. Sci. Comput. 40 (2009) 391-418. 